\title{
DIFFERENTIAL-DIFFERENCE OPERATORS ASSOCIATED TO REFLECTION GROUPS
}

\author{
CHARLES F. DUNKL
}

\begin{abstract}
There is a theory of spherical harmonics for measures invariant under a finite reflection group. The measures are products of powers of linear functions, whose zero-sets are the mirrors of the reflections in the group, times the rotation-invariant measure on the unit sphere in $\mathbf{R}^{n}$. A commutative set of differential-difference operators, each homogeneous of degree -1 , is the analogue of the set of first-order partial derivatives in the ordinary theory of spherical harmonics. In the case of $\mathbf{R}^{2}$ and dihedral groups there are analogues of the Cauchy-Riemann equations which apply to Gegenbauer and Jacobi polynomial expansions.
\end{abstract}

The analysis of orthogonality structures for polynomials in several variables is a problem of vast dimensions. This paper is part of an ongoing program to establish a workable theory for one particular class. The underlying structure is based on finite Coxeter groups: these are finite groups acting on Euclidean space, generated by reflections in the zero sets of a collection of linear functions (the "roots"); the weight functions for the orthogonality are products of powers of these linear functions restricted to the surface of the unit sphere. In addition, the weight function is required to be invariant under the action of the group. The resulting theory has strong similarities to the theory of spherical harmonics; this was established in previous papers of the author $[3,4,5]$. Most notably, a homogeneous polynomial is orthogonal to all polynomials of lower degree if and only if it is annihilated by a certain second-order differential-difference operator. Ordinary partial differentiation acts as an endomorphism on ordinary harmonic functions; the use of such operators and their adjoints leads to recurrence formulas and orthogonal decompositions for harmonic polynomials.

In this paper we construct a commutative set of first-order differentialdifference operators associated to the second-order operator previously mentioned.

Received by the editors June 1, 1987.

1980 Mathematics Subject Classification (1985 Revision). Primary 33A45, 33A65, 20H15; Secondary $20 \mathrm{C} 30,42 \mathrm{C} 10,51 \mathrm{~F} 15$.

Key words and phrases. Orthogonal polynomials in several variables, reflection groups, spherical harmonics, Gegenbauer polynomials, Jacobi polynomials.

During the preparation of this paper the author was partially supported by NSF Grant DMS86-01670. 
The main topics are the definition and the commutativity proof, the determination of the adjoints (the multivariable analogue of the three-term recurrence for one-variable orthogonal polynomials), and a study of the Cauchy-Riemann equations associated to dihedral groups. The latter topic includes conjugate series of Gegenbauer or Jacobi polynomial types.

Here is an outline of the contents of each section:

1. background on reflection groups and orthogonal polynomials, definition of the operators, commutativity, the Laplacian as a sum of squares;

2. determination of the adjoints, a fundamental selfadjoint operator, the relation of its eigenvalues to the character table of the reflection groups, degenerate parameter values;

3. the Cauchy-Riemann equations for a dihedral group, polynomials of Gegenbauer and Jacobi type.

Some of the results of this paper were announced in a talk at ICM 86Berkeley.

\section{BACKGROUND AND FUNDAMENTAL RESULTS}

We begin with basic facts about reflections and the groups they generate. For a nonzero vector $v \in \mathbf{R}^{N}$ define the reflection $\sigma_{v} \in O(N)$ (the orthogonal group) by

$$
x \sigma_{v}:=x-\left(2\langle x, v\rangle /|v|^{2}\right) v, \quad x \in \mathbf{R}^{N},
$$

where $\langle x, v\rangle:=\sum_{j=1}^{N} x_{j} v_{j}$, the inner product, and $|v|^{2}:=\langle v, v\rangle$. Thus, $v \sigma_{v}=$ $-v$ and $x \sigma_{v}=x$ if and only if $\langle x, v\rangle=0$. Any set of reflections generates a subgroup of $O(N)$, which is finite under certain conditions, established by Coxeter [1]. In this case such a group is called a finite reflection or Coxeter group.

Suppose now that $G$ is a Coxeter group with the set $\left\{\sigma_{i}: 1 \leq i \leq m\right\}$ of reflections (the list of all reflections in $G$ ). Choose a set of vectors $\left\{v_{i}: 1 \leq\right.$ $i \leq m\} \subset \mathbf{R}^{N}$ such that $\sigma_{i}=\sigma_{v_{i}}$ for $1 \leq i \leq m$, and $\left|v_{i}\right|=\left|v_{j}\right|$ whenever $\sigma_{i} \sim \sigma_{j}\left(\sigma_{i}\right.$ is conjugate to $\sigma_{j}$ in $G$; this implies $v_{i} w= \pm v_{j}$ for some $w \in G$, since $w^{-1} \sigma_{v} w=\sigma_{v w}$ for $\left.v \neq 0\right)$. Next we choose positive parameters $\alpha_{i}, 1 \leq$ $i \leq m$ such that $\alpha_{i}=\alpha_{j}$ whenever $\sigma_{i} \sim \sigma_{j}$. (In purely algebraic formulas the positivity restriction can be dropped, but there are still excluded "degenerate" values, to be discussed in $\S 2$.)

Define $h(x)=\prod_{j=1}^{m}\left|\left\langle x, v_{j}\right\rangle\right|^{\alpha_{j}}$; a $G$-invariant function (we will use " $h$ " as a short-hand notation for the collection of particular values of $v_{j}, \alpha_{j}$ ).

Let the sphere $S:=\left\{x \in \mathbf{R}^{N}:|x|=1\right\}$ with normalized rotation-invariant measure $d \omega$. Let $\nabla$ denote the gradient vector, and $\Delta:=\sum_{i=1}^{N}\left(\partial / \partial x_{i}\right)^{2}$, the Laplacian. 
1.1 Definition [5]. The $h$-Laplacian $\Delta_{h}$ is given by

$$
\Delta_{h} f(x):=\Delta f(x)+\sum_{j=1}^{m} \alpha_{j}\left[\frac{2\left\langle\nabla f(x), v_{j}\right\rangle}{\left\langle x, v_{j}\right\rangle}-\left|v_{j}\right|^{2} \frac{f(x)-f\left(x \sigma_{j}\right)}{\left\langle x, v_{j}\right\rangle^{2}}\right] .
$$

It was shown in [5, Proposition 1.3] that $\Delta_{h}$ is an endomorphism on the space of polynomials and is homogeneous of degree -2 . In analogy to harmonic polynomials the key result is

1.2 Theorem [5]. If $p$ is a homogeneous polynomial, then

$$
\int_{S} p q h^{2} d \omega=0
$$

for all polynomials $q$ with degree $(q)<$ degree $(p)$ if and only if $\Delta_{h} p=0$.

Polynomials in $\operatorname{ker} \Delta_{h}$ are called $h$-harmonic. Continuing the analogy to $\left(\partial / \partial x_{j}\right) \Delta=\Delta\left(\partial / \partial x_{j}\right)$ we construct a commutative set of first-order differentialdifference operators $\left\{T_{i}: 1 \leq i \leq N\right\}$ such that $\Delta_{h}=\sum_{i} T_{i}^{2}$ and $\Delta_{h} T_{j}=T_{j} \Delta_{h}$. Thus, each $T_{j}$ is an endomorphism on the $h$-harmonic polynomials. (The functions we use in the sequel are mostly polynomial; but the formulas hold in the presence of adequate differentiability.)

1.3 Definition. The $h$-gradient $\nabla_{h}$ is given by

$$
\nabla_{h} f(x):=\nabla f(x)+\sum_{j=1}^{m} \alpha_{j} \frac{f(x)-f\left(x \sigma_{j}\right)}{\left\langle x, v_{j}\right\rangle} v_{j} .
$$

When $f$ is polynomial, then so is $\left(f(x)-f\left(x \sigma_{j}\right)\right) /\left\langle x, v_{j}\right\rangle$; these operators have been used in the Schubert calculus (see Hiller [6]) of Coxeter groups. Without loss of generality, we will assume $\left|v_{j}\right|=1$ for all $j$ in the rest of this section.

The group $G$ acts on functions on $\mathbf{R}^{N}$ by the right regular representation $R(w) f(x):=f(x w),\left(x \in \mathbf{R}^{N}, w \in G\right)$. The action of $R$ on $\nabla_{h}$ is $\nabla_{h}(R(w) f)(x)=\left(\nabla_{h} f(x w)\right) w^{-1}$; the straightforward proof of this uses the fact that $\sigma_{j} w=w \sigma_{i}$ implies $\alpha_{i}=\alpha_{j}$.

1.4 Definition. For $u \in \mathbf{R}^{N}$ with $u \neq 0$ let $T_{u} f:=\left\langle\nabla_{h} f, u\right\rangle$; for $1 \leq i \leq N$ let $T_{i} f:=\left\langle\nabla_{h} f, e_{i}\right\rangle$ where $e_{i}$ is the standard unit vector of index $i,(0 \ldots 1 \ldots 0)$.

Before we prove commutativity we derive some identities needed in the proof. We let $\tilde{\sigma}_{i}$ denote the difference operator,

$$
\tilde{\sigma}_{i} f(x):=\left(f(x)-f\left(x \sigma_{i}\right)\right) /\left\langle x, v_{i}\right\rangle,
$$

associated to the reflection $\sigma_{i} \in G$. 
The following identities are straightforward (identity (1.6) uses the fact, $u-$ $\left.u \sigma_{j}=2\left\langle u, v_{j}\right\rangle v_{j}\right):$

$$
\begin{aligned}
\tilde{\sigma}_{i} \tilde{\sigma}_{j} f(x)= & \frac{f(x)}{\left\langle x, v_{i}\right\rangle\left\langle x, v_{j}\right\rangle}-\frac{f\left(x \sigma_{j}\right)}{\left\langle x, v_{i}\right\rangle\left\langle x, v_{j}\right\rangle} \\
& -\frac{f\left(x \sigma_{i}\right)}{\left\langle x, v_{i}\right\rangle\left\langle x, v_{j} \sigma_{i}\right\rangle}+\frac{f\left(x \sigma_{i} \sigma_{j}\right)}{\left\langle x, v_{i}\right\rangle\left\langle x, v_{j} \sigma_{i}\right\rangle},
\end{aligned}
$$

and $\tilde{\sigma}_{i}^{2}=0$;

$$
\begin{aligned}
& \left\langle\nabla \tilde{\sigma}_{j} f(x), u\right\rangle-\tilde{\sigma}_{j}\langle\nabla f(x), u\rangle \\
& \quad=\left(2\left\langle v_{j}, \nabla f\left(x \sigma_{j}\right)\right\rangle-\tilde{\sigma}_{j} f(x)\right)\left\langle u, v_{j}\right\rangle /\left\langle x, v_{j}\right\rangle .
\end{aligned}
$$

1.7 Proposition. Suppose $B(x, y)$ is a bilinear form on $\mathbf{R}^{N}$ such that $B\left(x \sigma_{v}, y \sigma_{v}\right)=B(y, x)$ whenever $v \in \operatorname{span}(x, y)$, and let $w$ be a plane rotation in $G$ (that is, $w$ is a product of two reflections and $w \neq e$, the identity), then:

(1) $\sum\left\{B\left(v_{i}, v_{j}\right) \alpha_{i} \alpha_{j} /\left\langle x, v_{i}\right\rangle\left\langle x, v_{j}\right\rangle: \sigma_{i} \sigma_{j}=w\right\}=0$ (as a rational function in $x)$;

(2) $\sum\left\{\alpha_{i} \alpha_{j} B\left(v_{i}, v_{j}\right) \tilde{\sigma}_{i} \tilde{\sigma}_{j}: \sigma_{i} \sigma_{j}=w\right\}=0$ (as an operator).

Proof. Let $E$ be the plane of $w$ (the orthogonal complement of the fixed point set). Thus $\sigma_{i} \sigma_{j}=w$ implies $v_{i}, v_{j} \in E$. Let $G_{1}$ be the subgroup of $G$ generated by $\left\{\sigma_{i}: v_{i} \in E\right\}$, (note $\sigma_{j} \in G_{1}$ implies $\sigma_{j} w \sigma_{j}=w^{-1}$ ), and let $m_{1}$ be the cardinality of the set of reflections in $G_{1}$. Denote the sum in (1) by $s(x)$. We will show $s\left(x \sigma_{l}\right)=s(x)$ for $\sigma_{l} \in G_{1}$, hence that $s$ is invariant under $G_{1}$.

Fix $\sigma_{l} \in G_{1}$ and define the functions $\varepsilon(i), \pi(i)$ by $\sigma_{l} \sigma_{i} \sigma_{l}=\sigma_{\pi(i)}$ and $v_{i} \sigma_{l}=$ $\varepsilon(i) v_{\pi(i)}$; thus $\varepsilon(i)= \pm 1, \pi$ is an involutory permutation, and $\varepsilon(\pi(i))=\varepsilon(i)$, for each $i, 1 \leq i \leq m$.

Then

$$
\begin{aligned}
s\left(x \sigma_{l}\right) & =\sum\left\{\frac{\alpha_{i} \alpha_{j} B\left(v_{i}, v_{j}\right)}{\left\langle x \sigma_{l}, v_{i}\right\rangle\left\langle x \sigma_{l}, v_{j}\right\rangle}: \sigma_{i} \sigma_{j}=w\right\} \\
& =\sum\left\{\alpha_{\pi(i)} \alpha_{\pi(j)} \frac{B\left(v_{\pi(i)}, v_{\pi(j)}\right)}{\left\langle x, v_{\pi(i)} \sigma_{l}\right\rangle\left\langle x, v_{\pi(j)} \sigma_{l}\right\rangle}: \sigma_{\pi(i)} \sigma_{\pi(j)}=w\right\} \\
& =\sum\left\{\alpha_{i} \alpha_{j} \frac{B\left(\varepsilon(i) v_{i} \sigma_{l}, \varepsilon(j) v_{j} \sigma_{l}\right)}{\left\langle x, v_{i}\right\rangle\left\langle x, v_{j}\right\rangle \varepsilon(i) \varepsilon(j)}: \sigma_{l} \sigma_{i} \sigma_{j} \sigma_{l}=w\right\} \\
& =\sum\left\{\alpha_{i} \alpha_{j} \frac{B\left(v_{j}, v_{i}\right)}{\left\langle x, v_{i}\right\rangle\left\langle x, v_{j}\right\rangle}: \sigma_{i} \sigma_{j}=\sigma_{l} w \sigma_{l}\right\} .
\end{aligned}
$$

We first replaced indices $(i, j)$ by $(\pi(i), \pi(j))$, then used $\alpha_{\pi(i)}=\alpha_{i}$ and the hypothesis on $B$. Because $G_{1}$ acts on a plane, $\sigma_{i} \sigma_{j}=\sigma_{l} w \sigma_{l}=w^{-1}$ if and only 
if $\sigma_{j} \sigma_{i}=w$, and so the last sum is $s(x)$. Finally

$$
s(x) \prod\left\{\left\langle x, v_{j}\right\rangle: v_{j} \in E\right\}
$$

is a polynomial of degree $\leq m_{1}-2$ and is alternating for $G_{1}$, hence must be 0 (see Coxeter and Moser [1, Chapter 9]).

For part (2), we begin with identity (1.5) and consider separately the coefficients of $f(x), f\left(x \sigma_{l}\right)$ and $f(x w)$. The coefficient of $f(x)$ is precisely $s(x)$ from part (1). For a fixed $\sigma_{l} \in G_{1}$, the coefficient of $f\left(x \sigma_{l}\right)$ is

$$
\frac{\alpha_{i} \alpha_{l} B\left(v_{i}, v_{l}\right)}{\left\langle x, v_{i}\right\rangle\left\langle x, v_{l}\right\rangle}+\frac{\alpha_{l} \alpha_{j} B\left(v_{l}, v_{j}\right)}{\left\langle x, v_{l}\right\rangle\left\langle x, v_{j} \sigma_{l}\right\rangle}
$$

where $\sigma_{i} \sigma_{l}=w=\sigma_{l} \sigma_{j}$. In terms of $\varepsilon, \pi$ as above, $j=\pi(i)$ and the second term becomes

$$
\alpha_{l} \alpha_{\pi(i)} B\left(v_{l}, \varepsilon(i) v_{i} \sigma_{l}\right) /\left(\left\langle x, v_{l}\right\rangle \varepsilon(i)\left\langle x, v_{i}\right\rangle\right)=\alpha_{l} \alpha_{i} B\left(v_{i}, v_{l} \sigma_{l}\right) /\left(\left\langle x, v_{l}\right\rangle\left\langle x, v_{i}\right\rangle\right) .
$$

But $v_{l} \sigma_{l}=-v_{l}$, and so the required coefficient is zero.

The coefficient of $f(x w)$ is

$$
\sum\left\{\alpha_{i} \alpha_{j} B\left(v_{i}, v_{j}\right) /\left(\left\langle x, v_{i}\right\rangle\left\langle x, v_{j} \sigma_{i}\right\rangle\right): \sigma_{i} \sigma_{j}=w\right\} .
$$

For a fixed $\sigma_{i} \in G_{1}$ there is a unique $\sigma_{j} \in G_{1}$ with $\sigma_{i} \sigma_{j}=w$. Let $\sigma_{k}=\sigma_{i} \sigma_{j} \sigma_{i}$ and $v_{k}=\varepsilon v_{j} \sigma_{i}$. The $(i, j)$-term in the sum equals

$$
\alpha_{i} \alpha_{j} \frac{B\left(v_{j} \sigma_{i}, v_{i} \sigma_{i}\right)}{\left\langle x, v_{i}\right\rangle\left\langle x, \varepsilon v_{k}\right\rangle}=-\alpha_{i} \alpha_{k} \frac{B\left(v_{k}, v_{i}\right)}{\left\langle x, v_{k}\right\rangle\left\langle x, v_{i}\right\rangle} .
$$

Further $\sigma_{k} \sigma_{i}=w$; thus the sum equals $-s(x)$ from part (1).

1.8 Corollary. Let $B(x, y)$ satisfy the hypothesis of Proposition 1.7, then

$$
\sum_{i, j=1}^{m} \alpha_{i} \alpha_{j} B\left(v_{i}, v_{j}\right) \tilde{\sigma}_{i} \tilde{\sigma}_{j}=0,
$$

as an operator.

Proof. The terms in the sum with $i=j$ are zero because each $\tilde{\sigma}_{i}^{2}=0$. The other terms are grouped together by the value of $\sigma_{i} \sigma_{j}$ (a plane rotation in $G$ ). Each group of terms sums to zero by 1.7, part (2).

1.9 Theorem. For nonzero $u, v \in \mathbf{R}^{N}, T_{u} T_{v}=T_{v} T_{u}$.

Proof. Expand $\left(T_{u} T_{v}-T_{v} T_{u}\right) f(x)=E_{1}+E_{2}+E_{3}$, where

$$
\begin{aligned}
E_{1}= & \langle\nabla\langle v, \nabla f(x)\rangle, u\rangle-\langle\nabla\langle u, \nabla f(x)\rangle, v\rangle=0, \\
E_{2}= & \sum_{j=1}^{m} \alpha_{j}\left\langle v, v_{j}\right\rangle\left(\left\langle\nabla \tilde{\sigma}_{j} f(x), u\right\rangle-\tilde{\sigma}_{j}\langle\nabla f(x), u\rangle\right) \\
& -\sum_{j=1}^{m} \alpha_{j}\left\langle u, v_{j}\right\rangle\left(\left\langle\nabla \tilde{\sigma}_{j} f(x), v\right\rangle-\tilde{\sigma}_{j}\langle\nabla f(x), v\rangle\right),
\end{aligned}
$$


and

$$
E_{3}=\sum_{i, j=1}^{m} \alpha_{i} \alpha_{j} B\left(v_{i}, v_{j}\right) \tilde{\sigma}_{i} \tilde{\sigma}_{j} f(x),
$$

with the form $B(x, y):=\langle u, x\rangle\langle v, y\rangle-\langle u, y\rangle\langle v, x\rangle$. Now $B$ satisfies the hypothesis of Corollary 1.8 , and thus $E_{3}=0$.

By formula (1.6),

$$
\begin{aligned}
E_{2}= & \sum_{j=1}^{m} \alpha_{j}\left(\left\langle v, v_{j}\right\rangle\left\langle u, v_{j}\right\rangle-\left\langle u, v_{j}\right\rangle\left\langle v, v_{j}\right\rangle\right) \\
& \times\left(2\left\langle v_{j}, \nabla f\left(x \sigma_{j}\right)\right\rangle-\tilde{\sigma}_{j} f(x)\right) /\left\langle x, v_{j}\right\rangle=0 .
\end{aligned}
$$

1.10 Theorem. Let $u_{1}, u_{2}, \ldots, u_{N}$ be an orthonormal basis of $\mathbf{R}^{N}$, then

$$
\sum_{j=1}^{N} T_{u_{j}}^{2}=\Delta_{h}
$$

Proof. For $u \neq 0$,

$$
\begin{aligned}
T_{u}^{2} f(x)= & \langle\nabla\langle\nabla f(x), u\rangle, u\rangle+2 \sum_{i=1}^{m} \alpha_{i}\left\langle u, v_{i}\right\rangle\langle u, \nabla f(x)\rangle /\left\langle x, v_{i}\right\rangle \\
& -\sum_{i=1}^{m} \alpha_{i}\left\langle u, v_{i}\right\rangle^{2}\left(f(x)-f\left(x \sigma_{i}\right)\right) /\left\langle x, v_{i}\right\rangle^{2} \\
& -2 \sum_{i=1}^{m} \alpha_{i}\left\langle u, v_{i}\right\rangle\left(\left\langle u, \nabla f\left(x \sigma_{i}\right)\right\rangle-\left\langle u, v_{i}\right\rangle\left\langle v_{i}, \nabla f\left(x \sigma_{i}\right)\right\rangle\right) /\left\langle x, v_{i}\right\rangle \\
& +\sum_{i, j=1}^{m} \alpha_{i} \alpha_{j}\left\langle u, v_{i}\right\rangle\left\langle u, v_{j}\right\rangle \tilde{\sigma}_{i} \tilde{\sigma}_{j} f(x) .
\end{aligned}
$$

This expansion uses identity (1.6), and the fact $u+u \sigma_{i}=2 u-2\left\langle u, v_{i}\right\rangle v_{i}$. Now sum $T_{u_{l}}^{2}$ and use the Parseval identity $\sum_{l}\left\langle u_{l}, u\right\rangle\left\langle u_{l}, v\right\rangle=\langle u, v\rangle$ to obtain

$$
\begin{aligned}
\sum_{l=1}^{N} T_{u_{l}}^{2} f(x)= & \Delta f(x)+\sum_{i=1}^{m} \alpha_{i}\left(2\left\langle v_{i}, \nabla f(x)\right\rangle /\left\langle x, v_{i}\right\rangle\right. \\
& \left.-\left(f(x)-f\left(x \sigma_{i}\right)\right) /\left\langle x, v_{i}\right\rangle^{2}\right) \\
& -2 \sum_{i=1}^{m} \alpha_{i}\left(\left\langle v_{i}, \nabla f\left(x \sigma_{i}\right)\right\rangle-\left\langle v_{i}, \nabla f\left(x \sigma_{i}\right)\right\rangle\right) /\left\langle x, v_{i}\right\rangle \\
& +\sum_{i, j} \alpha_{i} \alpha_{j}\left\langle v_{i}, v_{j}\right\rangle \tilde{\sigma}_{i} \tilde{\sigma}_{j} f(x)=\Delta_{h} f(x) .
\end{aligned}
$$

The last sum is zero by Corollary 1.8 applied to the form $B(x, y)=\langle x, y\rangle$.

1.11 Corollary. If $p$ is an h-harmonic polynomial, then so is $T_{u} p$, for $u \neq 0$, $u \in \mathbf{R}^{N}$.

The operators $T_{i}$ provide a method for establishing identities holding among $h$-harmonic polynomials. The following problem arises: given an $N$-tuple of 
polynomials $\left(p_{j}\right)_{j=1}^{N}$ with $T_{i} p_{j}=T_{j} p_{i}$ for each $i, j$ and $\sum_{i=1}^{N} T_{i} p_{i}=0$, can one find an $h$-harmonic polynomial $p$ with $p_{i}=T_{i} p$, each $i$ ? We leave this for later work.

\section{ADJOINTS AND RELATED OPERATORS}

We will determine the effect of $T_{i}^{*}$ on the $h$-harmonic polynomials with respect to the inner product structure of $L^{2}\left(S, h^{2} d \omega\right)$. We will also study the positive operator $\sum_{i=1}^{N} T_{i}^{*} T_{i}$ and its eigenvalues.

Let $P_{n}$ denote the space of $h$-harmonic polynomials which are homogeneous of degree $n$. By Theorem 1.2, $P_{n} \perp P_{m}$ for $n \neq m$. Since $T_{i} P_{n} \subset P_{n-1}$ we see that $T_{i}^{*} P_{n} \subset P_{n+1}$. It turns out that $T_{i}^{*}$ is close to being multiplication by $x_{i}$.

For the rest of this section, let $\gamma:=\sum_{i=1}^{m} \alpha_{i}$, the degree of $h$.

2.1 Theorem. If $f \in P_{n}$, then

$$
T_{i}^{*} f(x)=(N+2 n+2 \gamma)\left(x_{i} f(x)-(N+2 n+2 \gamma-2)^{-1}|x|^{2} T_{i} f(x)\right) .
$$

We proceed in several stages to prove this result. In the form

$$
x_{i} f(x)=(N+2 n+2 \gamma)^{-1} T_{i}^{*} f(x)+(N+2 n+2 \gamma-2)^{-1}|x|^{2} T_{i} f(x) \text {, }
$$

this is an analogue of the three-term recurrence for orthogonal polynomials of one variable.

2.2 Proposition. $\Delta_{h}\left(x_{i} f(x)\right)=x_{i} \Delta_{h} f(x)+2 T_{i} f(x), 1 \leq i \leq N$.

Proof. By the product rules for $\Delta$ and $\nabla$ we obtain

$$
\begin{aligned}
& \Delta_{h}\left(x_{i} f(x)\right)=x_{i} \Delta f(x)+2 \frac{\partial f}{\partial x_{i}} \\
& \quad+\sum_{j=1}^{m} \alpha_{j}\left[\frac{2\left\langle v_{j}, \nabla f\right\rangle x_{i}}{\left\langle v_{j}, x\right\rangle}+\frac{2 f(x)\left(v_{j}\right)_{i}}{\left\langle v_{j}, x\right\rangle}-\left|v_{j}\right|^{2} \frac{x_{i} f(x)-\left(x \sigma_{j}\right)_{i} f\left(x \sigma_{j}\right)}{\left\langle v_{j}, x\right\rangle^{2}}\right] \\
& =x_{i} \Delta_{h} f(x)+2 T_{i} f(x) ;
\end{aligned}
$$

in the last term

$$
x_{i} f(x)-\left(x \sigma_{j}\right)_{i} f\left(x \sigma_{j}\right)=x_{i}\left(f(x)-f\left(x \sigma_{j}\right)\right)+\left(x_{i}-\left(x \sigma_{j}\right)_{i}\right) f\left(x \sigma_{j}\right),
$$

and

$$
x_{i}-\left(x \sigma_{j}\right)_{i}=2\left\langle x, v_{j}\right\rangle\left(v_{j}\right)_{i} /\left|v_{j}\right|^{2} .
$$

2.3 Proposition. If $f \in P_{n}$ then

$$
x_{i} f-(N+2 n+2 \gamma-2)^{-1}|x|^{2} T_{i} f \in P_{n+1} .
$$

Proof. By Lemma 1.9 of [5],

$$
\Delta_{h}\left(|x|^{2} T_{i} f\right)=4(n+\gamma-1+N / 2) T_{i} f+|x|^{2} \Delta_{h} T_{i} f .
$$

Thus

$$
\Delta_{h}\left(x_{i} f-c|x|^{2} T_{i} f\right)=x_{i} \Delta_{h} f+(2-4(n+\gamma-1+N / 2) c) T_{i} f=0
$$


when $c$ has the appropriate value; since

$$
\Delta_{h} T_{i} f=T_{i} \Delta_{h} f=0 \text {. }
$$

2.4 Lemma. If $f$ is a homogeneous polynomial of degree $k$, then

$$
\int_{S} \frac{\partial f(x)}{\partial x_{i}} d \omega(x)=(N+k-1) \int_{S} x_{i} f(x) d \omega(x) .
$$

Proof. By the use of polar coordinates

$$
\int_{|x| \leq 1} g(x) d x=c_{N} \int_{0}^{1} r^{N-1} d r \int_{S} g(r x) d \omega(x),
$$

for some constant $c_{N}$, and each continuous function $g$ on the ball. Set $g(x)=$ $\left(\partial f(x) / \partial x_{i}\right)\left(1-|x|^{2}\right)$ and use integration by parts to obtain

$$
\begin{aligned}
\int_{S} \frac{\partial f}{\partial x_{i}} d \omega & =A_{1} \int_{|x| \leq 1} \frac{\partial f(x)}{\partial x_{i}}\left(1-|x|^{2}\right) d x \\
& =-A_{1} \int_{|x| \leq 1} f(x) \frac{\partial}{\partial x_{i}}\left(1-|x|^{2}\right) d x \\
& =2 A_{1} \int_{|x| \leq 1} x_{i} f(x) d x=\left(2 A_{1} / A_{2}\right) \int_{S} x_{i} f(x) d \omega(x),
\end{aligned}
$$

where

$$
A_{1}=\left(c_{N} \int_{0}^{1} r^{k+N-2}\left(1-r^{2}\right) d r\right)^{-1}
$$

and

$$
A_{2}=\left(c_{N} \int_{0}^{1} r^{k+N} d r\right)^{-1}
$$

thus

$$
2 A_{1} / A_{2}=N+k-1 \text {. }
$$

Proof of Theorem 2.1. Let $f \in P_{n+1}, g \in P_{n}$; then

$$
\begin{aligned}
\int_{S}\left(f T_{i} g\right. & \left.+g T_{i} f\right) h^{2} d \omega \\
= & \int_{S}\left(f \frac{\partial g}{\partial x_{i}}+g \frac{\partial f}{\partial x_{i}}+2 \sum_{j=1}^{m} \alpha_{j} f(x) g(x) \frac{\left(v_{j}\right)_{i}}{\left\langle x, v_{j}\right\rangle}\right) h(x)^{2} d \omega(x) \\
& -\sum_{j=1}^{m} \alpha_{j}\left(v_{j}\right)_{i} \int_{S}\left(\left(f(x) g\left(x \sigma_{j}\right)+f\left(x \sigma_{j}\right) g(x)\right) /\left\langle x, v_{j}\right\rangle\right) h(x)^{2} d \omega(x) \\
= & \int_{S} \frac{\partial}{\partial x_{i}}\left(f(x) g(x) h(x)^{2}\right) d \omega(x),
\end{aligned}
$$

because

$$
\frac{\partial}{\partial x_{i}}\left(h(x)^{2}\right)=2 \sum_{j=1}^{m} \alpha_{j}\left(\left(\frac{\partial}{\partial x_{i}}\left\langle x, v_{j}\right\rangle\right) /\left\langle x, v_{j}\right\rangle\right) h(x)^{2}
$$


and the second integral is zero; indeed

$$
\begin{aligned}
\int_{S} \frac{f(x) g\left(x \sigma_{j}\right)}{\left\langle x, v_{j}\right\rangle} h(x)^{2} d \omega(x) & =\int_{S} \frac{f\left(x \sigma_{j}\right) g(x)}{\left\langle x \sigma_{j}, v_{j}\right\rangle} h\left(x \sigma_{j}\right)^{2} d \omega(x) \\
& =-\int_{S} \frac{f\left(x \sigma_{j}\right) g(x)}{\left\langle x, v_{j}\right\rangle} h(x)^{2} d \omega(x)
\end{aligned}
$$

(since $v_{j} \sigma_{j}=-v_{j}$ and $h(x)^{2}$ is $G$-invariant). It is assumed here that each $\alpha_{j} \geq 1$; analytic continuation will allow $\alpha_{j} \geq 0$.

By the lemma, the above expression equals

$$
(N+2 \gamma+2 n) \int_{S} x_{i} f(x) g(x) h(x)^{2} d \omega(x) .
$$

Rearrange the resulting identity to obtain

$$
\int_{S}\left(T_{i} f\right) g h^{2} d \omega=\int_{S} f\left((N+2 n+2 \gamma) x_{i} g\right) h^{2} d \omega-\int_{S} f\left(T_{i} g\right) h^{2} d \omega .
$$

The last term is zero since $T_{i} g \in P_{n-1} \perp P_{n+1}$. By Proposition 2.3,

$$
g_{i}(x):=(N+2 n+2 \gamma)\left(x_{i} g(x)-(N+2 n+2 \gamma-2)^{-1}|x|^{2} T_{i} g(x)\right)
$$

is in $P_{n+1}$ and satisfies

$$
\int_{S} f g_{i} h^{2} d \omega=\int_{S}\left(T_{i} f\right) g h^{2} d \omega,
$$

(note $\int_{S} f(x)|x|^{2} T_{i} g(x) h(x)^{2} d \omega(x)=0$ ).

We now have a collection of selfadjoint operators leaving each $P_{n}$ invariant, namely, the span of $T_{j}^{*} T_{j}$ and $T_{j} T_{j}^{*} ; 1 \leq j \leq N$. The problem of determining explicit eigenvector decompositions of such operators will be left for further research.

One particular linear combination is of fundamental importance.

\subsection{Proposition.}

$$
\sum_{i=1}^{N} T_{i}^{*} T_{i} f(x)=(N+2 n+2 \gamma-2) \sum_{i=1}^{N} x_{i} T_{i} f(x),
$$

and

$$
\sum_{i=1}^{N} x_{i} T_{i} f(x)=n f(x)+\sum_{j=1}^{m} \alpha_{j}\left(f(x)-f\left(x \sigma_{j}\right)\right)
$$

for $f \in P_{n}, n=0,1,2, \ldots$.

Proof. For $f \in P_{n}$,

$$
\sum_{i=1}^{N} T_{i}^{*} T_{i} f(x)=(N+2 n+2 \gamma-2) \sum_{i=1}^{N} x_{i} T_{i} f(x)-c|x|^{2} \sum_{i=1}^{N} T_{i}^{2} f(x),
$$

for some constant $c$; but $f$ is $h$-harmonic and so $\sum_{i} T_{i}^{2} f=0$. 
Further

$$
\begin{aligned}
\sum_{i=1}^{N} x_{i} T_{i} f(x)= & \sum_{i=1}^{N} x_{i} \frac{\partial}{\partial x_{i}} f(x) \\
& +\sum_{j=1}^{m} \alpha_{j}\left(f(x)-f\left(x \sigma_{j}\right)\right) \sum_{i=1}^{N} x_{i}\left(v_{j}\right)_{i} /\left\langle x, v_{j}\right\rangle .
\end{aligned}
$$

From examples one suspects that explicit formulas for recurrences, specific polynomials, squares of norms, etc., are rational functions of $\left(\alpha_{1}, \ldots, \alpha_{m}\right)$. It appears that the singularities correspond to zero eigenvalues of $\sum_{i=1}^{N} T_{i}^{*} T_{i}$ on $P_{n}$, with $n \geq 1$. Those values of $\left(\alpha_{1}, \ldots, \alpha_{m}\right)$ corresponding to such an eigenvalue will be called degenerate values. From the scalar factor we see immediately that $\sum_{j=1}^{m} \alpha_{j}=-n-N / 2$, for some $n=0,1,2, \ldots$, specifies a set of degenerate values.

The other degenerate values are related to the eigenvalues of the operator

$$
A_{h} f(x):=\sum_{j=1}^{m} \alpha_{j}\left(f(x)-f\left(x \sigma_{j}\right)\right) .
$$

The right regular representation $R$ of $G$ on polynomials extends to one of the group algebra $\mathbf{C} G$, which can be decomposed into irreducible homogeneous components. Let $V$ be such a component of dimension $k$, with a basis $\left\{f_{j}\right\}_{j=1}^{k}$ of polynomials such that

$$
R(w) f_{j}(x)=\sum_{i=1}^{k} f_{i}(x) T_{i j}(w), \quad 1 \leq j \leq k,
$$

so that $w \mapsto\left(T_{i j}(w)\right)$ is an irreducible representation of $G$. The element $\sum_{w \in G} c_{w} w \in \mathbf{C} G$ acts on $V$ by

$$
R\left(\sum_{w} c_{w} w\right) f_{j}(x)=\sum_{i} f_{i}(x)\left(\sum_{w} c_{w} T_{i j}(w)\right) .
$$

By the underlying hypothesis on $\left(\alpha_{1}, \ldots, \alpha_{m}\right), \varphi:=\sum_{j=1}^{m}\left(\alpha_{j} e-\alpha_{j} \sigma_{j}\right)$ is a central element of C $G$. By Schur's lemma $A_{h}=R(\varphi)$ acts on $V$ by scalar multiplication, say by $\lambda \in \mathbf{C}$. To find $\lambda$, consider the trace of $R(\varphi)$, indeed

$$
k \lambda=\sum_{j=1}^{m} \alpha_{j}\left(k-\chi\left(\sigma_{j}\right)\right)
$$

where

$$
\chi(w):=\sum_{j} T_{j j}(w)
$$

the character of $T$. Thus $\lambda=\sum_{j=1}^{m} \alpha_{j}\left(1-\chi\left(\sigma_{j}\right) / k\right)$. Since $\sigma_{j}^{2}=e$, the eigenvalues of $T\left(\sigma_{j}\right)$ must be \pm 1 , hence $\chi\left(\sigma_{j}\right)$ is real, and $\alpha_{j} \geq 0$ for each $j$ implies $\lambda \geq 0$. 
2.6 Example. For the hyperoctahedral group $W_{N}$ on $\mathbf{R}^{N}$, the Weyl group of type $B_{N}$, the weight function is

$$
h(x)=\left(\prod_{i=1}^{N}\left|x_{i}\right|\right)^{\alpha}\left(\prod_{1 \leq i<j \leq N}\left|x_{i}^{2}-x_{j}^{2}\right|\right)^{\beta} .
$$

There are two conjugacy classes of reflections, one class of size $N$ containing the sign-changes, (for example $\left.\left(x_{1}, x_{2}, \ldots, x_{N}\right) \sigma_{1}=\left(-x_{1}, x_{2}, \ldots, x_{N}\right)\right)$, the other of size $N(N-1)$ containing transpositions (for example: $\left(x_{1}, \ldots, x_{N}\right) \sigma_{12}=$ $\left(x_{2}, x_{1}, x_{3}, \ldots, x_{N}\right)$ and $\left.\left(x_{1}, \ldots, x_{N}\right) \sigma_{12}^{\prime}=\left(-x_{2},-x_{1}, x_{3}, \ldots, x_{N}\right)\right)$.

Recall that the irreducible characters $\chi_{\lambda}$ of the symmetric group $S_{k}$ on $k$ letters are determined by partitions $\lambda$; where $\lambda=\left(\lambda_{1}, \lambda_{2}, \ldots\right)$ with $\lambda_{1} \geq \lambda_{2} \geq$ $\cdots \geq \lambda_{k} \geq 0, \lambda_{j} \in Z_{+}$, and $|\lambda|:=\sum \lambda_{i}=k$.

For any ordered pair of partitions $(\lambda, \mu)$ with $|\lambda|+|\mu|=N$ there corresponds an irreducible character $\chi_{(\lambda, \mu)}$ of $W_{N}$; it is induced from the character $\left(w_{1}, w_{2}\right) \mapsto \chi_{\lambda}\left(\bar{w}_{1}\right) \chi_{\mu}\left(\bar{w}_{2}\right) \gamma\left(w_{2}\right)$ on $W_{|\lambda|} \times W_{|\mu|} \subset W_{N}$, (where $w \mapsto \bar{w}$ is the "sign-forgetting" homomorphism of $W_{k}$ onto $S_{k}$, considered as the group of $k \times k$ permutation matrices, and $\gamma(w)$ is the product of the $(-1)$ entries in the matrix $w$ ).

Lusztig [7] gives this description, as well as the Poincare series counting the appearances of $\chi_{(\lambda, \mu)}$ on spaces of homogeneous polynomials on $\mathbf{R}^{N}$. Here we only state the eigenvalues of $A_{h}$ on any subspace on which $\chi_{(\lambda, \mu)}$ is realized. By a formula of Young $[9, \mathrm{p} .282]$

$$
\chi_{(\lambda, \mu)}\left(\sigma_{i}\right) / \chi_{(\lambda, \mu)}(e)=1-2|\mu| / N
$$

and

$$
\chi_{(\lambda, \mu)}\left(\sigma_{12}\right) / \chi_{(\lambda, \mu)}(e)=\left(\sum_{i} \lambda_{i}\left(\lambda_{1}+1-2 i\right)+\sum_{j} \mu_{j}\left(\mu_{j}+1-2 j\right)\right) /(N(N-1)) \text {. }
$$

The latter formula involves $\chi_{\lambda}\left(\sigma_{12}\right) / \chi_{\lambda}(e)$ for characters of $S_{|\lambda|}$, (and similarly for $\mu)$. There is another informative expression: for a partition $\lambda$ let $\lambda^{\prime}$ be the associated partition, with transposed Ferrers diagram, then

$$
\sum_{i} \lambda_{i}\left(\lambda_{i}+1-2 i\right)=2\left(\sum_{i}\left(\begin{array}{c}
\lambda_{i} \\
2
\end{array}\right)-\sum_{j}\left(\begin{array}{c}
\lambda_{j}^{\prime} \\
2
\end{array}\right)\right) .
$$

2.7 Proposition. The eigenvalues of $A_{h}$ on the space of polynomials on which $W_{N}$ acts according to $\chi_{(\lambda, \mu)}$ is

$$
2|\mu| \alpha+2 \beta\left(\left(\begin{array}{c}
N \\
2
\end{array}\right)-\sum_{i}\left(\begin{array}{c}
\lambda_{i} \\
2
\end{array}\right)+\sum_{i}\left(\begin{array}{c}
\lambda_{i}^{\prime} \\
2
\end{array}\right)-\sum_{i}\left(\begin{array}{c}
\mu_{i} \\
2
\end{array}\right)+\sum_{i}\left(\begin{array}{c}
\mu_{i}^{\prime} \\
2
\end{array}\right)\right) .
$$




\section{THE DIHEDRAL GROUPS}

For reflection groups on $\mathbf{R}^{2}$, namely the dihedral groups, we use the factorization $\Delta_{h}=T_{1}^{2}+T_{2}^{2}=\left(T_{1}+i T_{2}\right)\left(T_{1}-i T_{2}\right)$ to produce the analogue of the Cauchy-Riemann equations. Muckenhoupt and Stein [8] proved some deep results about conjugate series for Gegenbauer expansions, and Gasper in an unpublished note (from 1970, private communication) discussed similar problems for Jacobi polynomial series.

Just as ordinary harmonic polynomials on $\mathbf{R}^{2} \cong \mathbf{C}$ are linear combinations of analytic polynomials (in the kernel of $\partial / \partial \bar{z}:=\frac{1}{2}(\partial / \partial x+i(\partial / \partial y))$ ) and their conjugates, the $h$-harmonic polynomials are in the span of the kernels of $T_{1} \pm$ $i T_{2}$, although these two kernels need not be orthogonal.

We will list the dihedral groups and the associated homogeneous polynomials in the kernel of $T_{1}+i T_{2}$. We will also determine the generalization of the formula $(\partial / \partial z) z^{n}=n z^{n-1}$ in each case.

3.1 Definition. For a reflection (dihedral) group $G$ on $\mathbf{R}^{2} \cong \mathbf{C}$ and associated weight function $h$, let $\bar{T}:=\frac{1}{2}\left(T_{1}+i T_{2}\right)$ and $T:=\frac{1}{2}\left(T_{1}-i T_{2}\right)$, (further notation: $\left.z=x_{1}+i x_{2}, \partial / \partial \bar{z}=\frac{1}{2}\left(\left(\partial / \partial x_{1}\right)+i\left(\partial / \partial x_{2}\right)\right),(\partial / \partial z)=\frac{1}{2}\left(\left(\partial / \partial x_{1}\right)-i\left(\partial / \partial x_{2}\right)\right)\right)$. Also let $K_{h}$ denote the kernel of $\bar{T}$. (We decline to introduce the term " $h$ analytic"!)

In complex terms the divided-difference terms are expressed as follows:

3.2 Lemma. Let $\omega \in \mathbf{C},|\omega|=1$, let $v:=(-\operatorname{Im} \omega, \operatorname{Re} \omega) \in \mathbf{R}^{2}$; then for a polynomial $f$ in $x$, or $(z, \bar{z})$,

$$
\frac{1}{2} \frac{f(x)-f\left(x \sigma_{v}\right)}{\langle x, v\rangle}(-\operatorname{Im} \omega+i \varepsilon \operatorname{Re} \omega)=\frac{f(z)-f\left(\bar{z} \omega^{2}\right)}{z-\bar{z} \omega^{2}} \xi,
$$

where $\xi=1$ for $\varepsilon=-1$ and $\xi=-\omega^{2}$ for $\varepsilon=1$, (terms in $T, \bar{T}$ respectively).

The dihedral group $D_{n}$, the group of symmetries of a regular $n$-gon, has one conjugacy class of reflections when $n$ is odd, and two classes when $n$ is even. By rotation of axes we can assume the real axis $\left(x_{2}=0\right)$ is one of the mirrors for $G$. Then

$$
h(z)=\left(\left(z^{k}-\bar{z}^{k}\right) / 2 i\right)^{\alpha}\left(\left(z^{k}+\bar{z}^{k}\right) / 2\right)^{\beta}
$$

is associated to $D_{2 k}$ if $\alpha, \beta>0$, and $D_{k}$ if $\alpha>0, \beta=0$.

Fix $k=1,2, \ldots$ and let $\omega:=e^{\pi i / k}$. By use of Lemma 3.2 and the factorizations

$$
z^{k}-\bar{z}^{k}=\prod_{j=0}^{k-1}\left(z-\bar{z} \omega^{2 j}\right) \quad \text { and } \quad z^{k}+\bar{z}^{k}=\prod_{j=0}^{k-1}\left(z-\bar{z} \omega^{2 j+1}\right) .
$$


We obtain the following formulas:

$$
\bar{T} f(z)=\frac{\partial f}{\partial \bar{z}}-\alpha \sum_{j=0}^{k-1} \frac{f(z)-f\left(\bar{z} \omega^{2 j}\right)}{z-\bar{z} \omega^{2 j}} \omega^{2 j}-\beta \sum_{j=0}^{k-1} \frac{f(z)-f\left(\bar{z} \omega^{2 j+1}\right)}{z-\bar{z} \omega^{2 j+1}} \omega^{2 j+1} .
$$

By the method of partial fractions we obtain the following identities, used in the sequel:

$$
\begin{gathered}
\sum_{j=0}^{k-1} \frac{1}{t-\omega^{2 j}}=\frac{k t^{k-1}}{t^{k}-1}, \\
\sum_{j=0}^{k-1} \frac{\omega^{2 j}}{t-\omega^{2 j}}=\frac{k}{t^{k}-1} \quad(t \in \mathbf{C}) .
\end{gathered}
$$

Most of the work in describing $K_{h}$ (the kernel of $\bar{T}$ ) reduces to the case $k=1$.

3.7 Proposition. Let $f(z)=g\left(z^{k}\right)$ and $\zeta:=z^{k}$, then

$$
\begin{aligned}
& T f(z)=k z^{k-1}\left(\frac{\partial g}{\partial \zeta}+\alpha \frac{g(\zeta)-g(\bar{\zeta})}{\zeta-\bar{\zeta}}+\beta \frac{g(\zeta)-g(-\bar{\zeta})}{\zeta+\bar{\zeta}}\right), \\
& \bar{T} f(z)=k \bar{z}^{k-1}\left(\frac{\partial g}{\partial \bar{\zeta}}-\alpha \frac{g(\zeta)-g(\bar{\zeta})}{\zeta-\bar{\zeta}}+\beta \frac{g(\zeta)-g(-\bar{\zeta})}{\zeta+\bar{\zeta}}\right) .
\end{aligned}
$$

Proof. Substitute $g$ in formulas (3.3) and (3.4), note

$$
\left(\bar{z} \omega^{2 j}\right)^{k}=\bar{z}^{k}=\bar{\zeta}, \quad\left(\bar{z} \omega^{2 j+1}\right)^{k}=-\bar{\zeta},
$$

and use (3.5) and (3.6) to evaluate the resulting sums, with $t=z / \bar{z}$, and $z /(\bar{z} \omega)$ respectively.

We will show that if $f(z)=g\left(z^{k}\right)$ and $\bar{T} f=0$ then $\bar{T}\left(z^{l} f(z)\right)=0$ for $0 \leq l \leq k-1$; this will allow the complete listing of homogeneous polynomials in $K_{h}$.

\subsection{Lemma.}

$$
\bar{T}(z f(z))=z \bar{T} f(z)-\alpha \sum_{j=0}^{k-1} \omega^{2 j} f\left(\bar{z} \omega^{2 j}\right)-\beta \omega \sum_{j=0}^{k-1} \omega^{2 j} f\left(\bar{z} \omega^{2 j+1}\right),
$$

for any polynomial $f$.

Proof. A typical term in $\bar{T}(z f(z))$ is of the form

$$
\begin{aligned}
& \omega^{j}\left(z f(z)-\bar{z} \omega^{j} f\left(\bar{z} \omega^{j}\right)\right) /\left(z-\bar{z} \omega^{j}\right) \\
& \quad=z \omega^{j}\left(f(z)-f\left(\bar{z} \omega^{j}\right)\right) /\left(z-\bar{z} \omega^{j}\right)+\omega^{j} f\left(\bar{z} \omega^{j}\right) .
\end{aligned}
$$


3.9 Proposition. Suppose $\bar{T} g\left(z^{k}\right)=0$ then $\bar{T}\left(z^{l} g\left(z^{k}\right)\right)=0$ for $0 \leq l \leq k-1$. Proof. Proceed by induction. Let $f(z)=z^{l} g\left(z^{k}\right)$ with $0 \leq l \leq k-2$ and assume $\bar{T} f=0$; then

$$
\bar{T}\left(z^{l+1} g\left(z^{k}\right)\right)=z \bar{T} f(z)-\left(\alpha g\left(\bar{z}^{k}\right) \bar{z}^{l}+\beta \omega \bar{z}^{l} g\left(-\bar{z}^{k}\right)\right) \sum_{j=0}^{k-1} \omega^{2 j(l+1)} .
$$

The latter sum is zero if $1 \leq l+1 \leq k-1$ since $\omega^{2}$ is a primitive $k$ th root of unity.

3.10 The group $D_{1}=Z_{2}$. Set $k=1, \beta=0$ to obtain $h(x)=x_{2}^{\alpha}$. In polar coordinates the measure is $\left(\sin ^{2} \theta\right)^{\alpha} d \theta$, and the orthogonal polynomials of degree $n$ are obviously $r^{n} C_{n}^{\alpha}(\cos \theta), r^{n}(\sin \theta) C_{n-1}^{\alpha+1}(\cos \theta)$ (see paragraph 4.1 in [5] for a detailed presentation), where $C_{n}^{\alpha}$ denotes the Gegenbauer polynomial of index $\alpha$ and degree $n$.

Some linear combination of these two must be in $K_{h}$ (the kernel of $\bar{T}$ ); indeed it is

$$
r^{n}\left(\frac{n+2 \alpha}{2 \alpha} C_{n}^{\alpha}(\cos \theta)+i \sin \theta C_{n-1}^{\alpha+1}(\cos \theta)\right) .
$$

There is a more elegant expression using the Heisenberg polynomials [2], which are given by the generating function

$$
\sum_{n=0}^{\infty} t^{n} C_{n}^{(\gamma, \delta)}(z)=(1-t \bar{z})^{-\gamma}(1-t z)^{-\delta}
$$

for $\gamma, \delta>0$. Thus

$$
C_{n}^{(\gamma, \delta)}(z)=\sum_{j=0}^{n} \frac{(\gamma)_{j}(\delta)_{n-j}}{j !(n-j) !} \bar{z}^{j} z^{n-j}, \quad z \in \mathbf{C}
$$

and

$$
C_{n}^{(\gamma, \gamma)}\left(r e^{i \theta}\right)=r^{n} C_{n}^{\gamma}(\cos \theta)
$$

3.11 Proposition. For

$$
h(x)=x_{2}^{\alpha}, \quad \bar{T} C_{n}^{(\alpha, \alpha+1)}(z)=0, \quad n \geq 0,
$$

and

$$
\begin{aligned}
C_{n}^{(\alpha, \alpha+1)}\left(r e^{i \theta}\right)= & r^{n}((n+2 \alpha) /(2 \alpha)) C_{n}^{\alpha}(\cos \theta) \\
& +i r^{n}(\sin \theta) C_{n-1}^{\alpha+1}(\cos \theta)
\end{aligned}
$$

in terms of Gegenbauer polynomials.

Proof. It suffices to show that

$$
\bar{T} \sum_{n=0}^{\infty} t^{n} C_{n}^{(\alpha, \alpha+1)}(z)=0 \quad(0<t<1,|z| \leq 1) .
$$


Indeed, from the generating function

$$
\begin{aligned}
\bar{T}(1-t \bar{z})^{-\alpha}(1-t z)^{-\alpha-1}=\alpha t(1-t \bar{z})^{-\alpha-1}(1-t z)^{-\alpha-1} \\
\quad-\alpha\left((1-t \bar{z})^{-\alpha}(1-t z)^{-\alpha-1}-(1-t z)^{-\alpha}(1-t \bar{z})^{-\alpha-1}\right) /(z-\bar{z}) \\
=\alpha(1-t \bar{z})^{-\alpha-1}(1-t z)^{-\alpha-1}(t-(1-t \bar{z}-1+t z) /(z-\bar{z}))=0 .
\end{aligned}
$$

The other claim comes from identity (10) on p. 704 of [2].

Since $\bar{T} T C_{n}^{(\alpha, \alpha+1)}(z)=0, T C_{n}^{(\alpha, \alpha+1)}$ must be a multiple of $C_{n-1}^{(\alpha, \alpha+1)}(z)$. The following are obtained by straightforward computation using well-known formulas.

$$
\begin{gathered}
T C_{n}^{(\alpha, \alpha+1)}(z)=(n+2 \alpha) C_{n-1}^{(\alpha, \alpha+1)}(z), \quad n \geq 1, \\
\int_{-\pi}^{\pi}\left|C_{n}^{(\alpha, \alpha+1)}\left(e^{i \theta}\right)\right|^{2}\left(\sin ^{2} \theta\right)^{\alpha} d \theta \\
=\frac{(2 \alpha+1)_{n}}{n !} 2 B\left(\alpha+\frac{1}{2}, \frac{1}{2}\right), \quad \text { (the beta function) } .
\end{gathered}
$$

3.14 The group $D_{2 m+1}$. Set $k=2 m+1$ and $\beta=0$. By Propositions 3.9 and 3.11 the homogeneous polynomial in $K_{h}$ of degree $(2 m+1) n+l, 0 \leq l \leq 2 m$ is $z^{l} C_{n}^{(\alpha, \alpha+1)}\left(z^{2 m+1}\right)$. The degenerate values (see Proposition 2.5 and its sequel) are easily shown to be $\alpha=-j /(2 m+1)$ and $\alpha=-j / 2$ for $j=1,2,3, \ldots$. Note that $z^{l} C_{n}^{(\alpha, \alpha+1)}\left(z^{2 m+1}\right)$ is orthogonal to its conjugate only when $1 \leq l \leq$ $2 m$, with respect to the measure $\left(\sin ^{2}(2 m+1) \theta\right)^{\alpha} d \theta$, in contrast to the ordinary case $(\alpha=0)$.

3.15 The group $D_{2}$. Set $k=1$ and $\alpha, \beta>0$.

In polar coordinates the corresponding measure on the circle is

$$
\left(\sin ^{2} \theta\right)^{\alpha}\left(\cos ^{2} \theta\right)^{\beta} d \theta=2^{-\alpha-\beta}(1-\cos 2 \theta)^{\alpha}(1+\cos 2 \theta)^{\beta} d \theta .
$$

Let

$$
\begin{aligned}
f_{2 n}(z):= & r^{2 n} P_{n}^{(\alpha-1 / 2, \beta-1 / 2)}(\cos 2 \theta) \\
& +(i / 2) r^{2 n}(\sin 2 \theta) P_{n-1}^{(\alpha+1 / 2, \beta+1 / 2)}(\cos 2 \theta), \quad n \geq 0, \\
f_{2 n+1}(z):= & r^{2 n}\left(\left(n+\alpha+\frac{1}{2}\right)(r \cos \theta) P_{n}^{(\alpha-1 / 2, \beta+1 / 2)}(\cos 2 \theta)\right. \\
& \left.+i\left(n+\beta+\frac{1}{2}\right)(r \sin \theta) P_{n}^{(\alpha+1 / 2, \beta-1 / 2)}(\cos 2 \theta)\right),
\end{aligned}
$$

where $z=r e^{i \theta}$ (and $\cos 2 \theta=\left(z^{2}+\bar{z}^{2}\right) /(2 z \bar{z})$, etc.), and $P_{n}^{(\gamma, \delta)}$ denotes the Jacobi polynomial of index $(\gamma, \delta)$ and degree $n$. Then $f_{2 n}$ and $f_{2 n+1}$ are (real-) homogeneous polynomials in $z, \bar{z}$; clearly the real and imaginary parts comprise an orthogonal basis of polynomials for the given measure. Gasper 
defined a version of the Cauchy-Riemann equations satisfied by $f_{2 n}$. By some tedious calculations.

$$
\begin{gathered}
\bar{T} f_{m}(z)=0, \quad m=0,1, \ldots, \\
T f_{2 n}=2 f_{2 n-1} \text { and } T f_{2 n+1}=2\left(n+\alpha+\frac{1}{2}\right)\left(n+\beta+\frac{1}{2}\right) f_{2 n}, \\
\int_{-\pi}^{\pi}\left|f_{2 n}\left(e^{i \theta}\right)\right|^{2} d \mu_{\alpha, \beta}(\theta)=\frac{\left(\alpha+\frac{1}{2}\right)_{n}\left(\beta+\frac{1}{2}\right)_{n}}{n !(\alpha+\beta+1)_{n}},
\end{gathered}
$$

and

$$
\int_{-\pi}^{\pi}\left|f_{2 n+1}\left(e^{i \theta}\right)\right|^{2} d \mu_{\alpha, \beta}(\theta)=\frac{\left(\alpha+\frac{1}{2}\right)_{n+1}\left(\beta+\frac{1}{2}\right)_{n+1}}{n !(\alpha+\beta+1)_{n}},
$$

where

$$
d \mu_{\alpha, \beta}(\theta):=\left(2 B\left(\alpha+\frac{1}{2}, \beta+\frac{1}{2}\right)\right)^{-1}\left(\sin ^{2} \theta\right)^{\alpha}\left(\cos ^{2} \theta\right)^{\beta} d \theta
$$

When

$$
\alpha=0=\beta, \quad f_{2 n}(z)=\left(\left(\frac{1}{2}\right)_{n} / n !\right) z^{2 n}
$$

and

$$
f_{2 n+1}(z)=\left(\left(\frac{1}{2}\right)_{n+1} / n !\right) z^{2 n+1} .
$$

3.19 The group $D_{2 m}$. Set $k=m$ and $\alpha, \beta>0$. By Proposition 3.9 the homogeneous polynomials in $K_{h}$ are (with $0 \leq l \leq m-1$ ):

$$
\begin{gathered}
z^{l} f_{2 n}\left(z^{m}\right), \quad \text { of degree } 2 n m+l ; \\
z^{l} f_{2 n+1}\left(z^{m}\right), \quad \text { of degree }(2 n+1) m+l .
\end{gathered}
$$

The degenerate values for $D_{2 m}$ are $\alpha+\beta=-j, \alpha+\beta=-j / m, \alpha=-1 / 2-j$, $\beta=-1 / 2-j$, for $j=1,2,3, \ldots$.

3.20 The action of $T$. An easy computation is possible by use of some identities.

Let (in the notation of (3.3) and (3.4))

$$
\Psi f(z):=\alpha \sum_{j=0}^{k-1} f\left(\bar{z} \omega^{2 j}\right)+\beta \sum_{j=0}^{k-1} f\left(\bar{z} \omega^{2 j+1}\right) .
$$

By Proposition 2.5, if $f$ is (real) homogeneous then

$$
(z T+\bar{z} \bar{T}) f(z)=\left(x_{1} T_{1}+x_{2} T_{2}\right) f(z)=(\operatorname{deg} f+k(\alpha+\beta)) f(z)-\Psi f(z) .
$$

Further, by an argument similar to that of Lemma 3.8,

$$
T(z f(z))=f(z)+z T f(z)+\Psi f(z) .
$$

Adding the two identities we obtain

$$
T(z f(z))=(1+\operatorname{deg} f+k(\alpha+\beta)) f(z)-\bar{z} \bar{T} f(z) .
$$

Now suppose $\bar{T} g\left(z^{k}\right)=0$ (as in Proposition 3.9), then

$$
\bar{T}\left(z^{l} g\left(z^{k}\right)\right)=0
$$


for $0 \leq l \leq k-1$, and

$$
T\left(z^{l} g\left(z^{k}\right)\right)=(l+k(\alpha+\beta+\operatorname{deg} g)) z^{l-1} g\left(z^{k}\right)
$$

for $1 \leq l \leq k-1$. The calculation of $\operatorname{Tg}\left(z^{k}\right)$ reduces to the case $k=1$ by use of Proposition 3.7. For example, on the group $D_{2 m}$

$$
T f_{2 n+1}\left(z^{m}\right)=2 m\left(n+\alpha+\frac{1}{2}\right)\left(n+\beta+\frac{1}{2}\right) z^{m-1} f_{2 n}\left(z^{m}\right)
$$

(in the notation of 3.15 and 3.19).

3.22 Concluding remarks. There is a maximum principle for $h$-harmonic polynomials (Theorem 2.10 of [5]); however, this does not seem to directly imply a maximum modulus principle for $K_{h}$. We also leave for further research the determination of explicit kernels of Cauchy or conjugate Poisson type.

\section{REFERENCES}

1. H. S. M. Coxeter and W. O. J. Moser, Generators and relations for discrete groups, SpringerVerlag, New York, Berlin and Heidelberg, 1957.

2. C. F. Dunkl, An addition theorem for Heisenberg harmonics, Conference on Harmonic Analysis in Honor of Antoni Zygmund, Wadsworth International, 1982, pp. 688-705.

3. __ Orthogonal polynomials on the sphere with octahedral symmetry, Trans. Amer. Math. Soc. 282 (1984), 555-575.

4. __ Orthogonal polynomials with symmetry of order three, Canad. J. Math. 36 (1984), 685717.

5. __ Reflection groups and orthogonal polynomials on the sphere, Math. Z. 197 (1988), 33-60.

6. H. Hiller, Geometry of Coxeter groups, Pitman, Boston, London and Melbourne, 1982.

7. G. Lusztig, Irreducible representations of finite classical groups, Invent. Math. 43 (1977), 125175.

8. B. Muckenhoupt and E. Stein, Classical expansions and their relation to conjugate harmonic functions, Trans. Amer. Math. Soc. 118 (1965), 17-92.

9. A. Young, On quantitative substitutional analysis. V, Proc. London Math. Soc. (2) 31 (1930), 273-288.

Department of Mathematics, University of Virginia, Charlottesville, Virginia 22903 